\title{
Transitions and Phases of Polytetrafluoroethylene (Teflon)'
}

\author{
Charles E. Weir
}

\begin{abstract}
Transitions in polytetrafluoroethylene (Teflon) have been studied as a function of pressure and temperature. A high-pressure transition (5,000 atm) and a lower pressure transition were studied. These are believed to result from three polymorphic forms. The pressure of the high-pressure transition decreases and that of the low-pressure transition increases with increasing temperature. A triple point is indicated at approximately $70^{\circ} \mathrm{C}$, and a third equilibrium line is found to proceed to higher pressures with increasing temperature from this point. Experimental errors involved render tests of all thermodynamic conditions at the triple point indeterminate.
\end{abstract}

\section{Introduction}

In the course of a continuing study of pressurevolume-temperature relationships in high-polymeric materials measurements have been made on polytetrafluoroethylene (Teflon). This report describes the pressure-temperature dependence of the transitions of Teflon. These transitions show a surprisingly complex behavior that is unique among all the polymers studied to date.

The room-temperature transition was discovered by Rigby and Bunn [1] ${ }^{2}$ and independently by the author [2] at about the same time. The transition that occurs at about $20^{\circ} \mathrm{C}$ is a reversible first-order transition producing reversible changes in the $\mathrm{X}$-ray diffraction pattern. The changes in the X-ray pattern have been interpreted as arising from specific changes in the crystal structure of the Teflon [1]. Quinn, Roberts, and Work [3] studied the transition extensively by volumetric methods and observed that the transition was complex, the complexity consisting of a double break in the volume-temperature curve associated with some degree of hysteresis. They evaluated the total volume change (that is, the sum of the two observed discontinuities) as 1.23 percent. Pierce, Bryant, and Whitney [4] have recently studied the X-ray pattern of Teflon over a wide temperature range.

Bridgman [5] and the author [6] have reported first-order transitions in Teflon under high pressures at room temperature. Bridgman reported the transition at $6,500 \mathrm{~kg} / \mathrm{cm}^{2}$ as involving a volume change of 2.26 percent, whereas the author found the transition centering about $5,500 \mathrm{~atm}$ at $25.5^{\circ} \mathrm{C}$, with an associated volume change of 2.35 percent. Differences between these data may well be due to variations in samples.

Finally, Furukawa [7] has measured the specificheat-temperature properties of Teflon. His data show the double discontinuity referred to, and he evaluated the total latent heat of the two breaks as approximately $8 \mathrm{j} / \mathrm{g}$. It is immediately apparent that this latent heat is not sufficiently large to fit the assumption that the high-pressure and room-temperature transitions are related. The 2:1 ratio of volume changes now tends to confirm this conclusion.

As a result of the present studies it is concluded

1 This paper is a preliminary summary of work on a project sponsored by the Office of Naval Research.

2 Figures in brackets refer to literature references at the end of this report. that Teflon undergoes two separate transitions, one at high and one at lower pressures at temperatures below $70^{\circ} \mathrm{C}$. The phase diagram for these transitions indicates at least three forms for the crystalline regions of Teflon. The evidence points to the fact that these are most probably polymorphic forms.

\section{Experimental Method}

The apparatus and experimental procedure have been described in detail previously [8] and will not be discussed fully here. Briefly, an experiment consists in forcing a leakproof piston into the bore of a heavywalled cylinder containing the specimen and the manganin pressure gage immersed in a suitable pressure transmitting medium, and taking pressure-volume readings. For locating a transition a graph of pressure versus piston position is constructed, the transition appearing as a discontinuity in this curve.

In these experiments the hydraulic press and pressure vessel were enclosed in a thermostatted air bath that could be maintained at elevated or reduced temperatures. Temperatures were measured with a calibrated mercury thermometer set in a well in a massive brass block fastened to and in intimate thermal contact with the pressure vessel. A maximum variation of $\pm 0.35 \mathrm{deg} C$ was noted on this thermometer at any temperature. However, the enormous heat capacity of the pressure vessel probably reduced the variation in temperature of the specimen to a considerably smaller value.

Inasmuch as the pressure gage was subjected to various temperatures in these experiments, it was calibrated at most temperatures used. Fixed points for calibration were taken from the International Critical Tables [9] but originate from data obtained by Bridgman. The transitions used for calibration were: Water-ice VI at lower temperatures; freezing of chloroform and $\mathrm{CCl}_{4}$ I to II at intermediate temperatures; and urea I to. III at higher temperatures. Small inconsistencies noted in these calibrations were not studied, the variations being of such small magnitude that any error resulting was negligible in these experiments. However, subjection of the gage to the elevated temperatures resulted in limiting the measurements to a maximum temperature of approximately $80^{\circ}$ C. After continued exposure to this temperature, marked drifting of the resistance of the pressure gage was observed. This drift is probably due to the action of the confining 
liquid on the shellac-treated gage coil. A study of the behavior of this or other materials to higher temperatures would require considerable modification of the apparatus to permit the pressure gage to remain at room temperature.

All data in these experiments were measured isothermally. Isobaric measurements were attempted, but the large heat capacity of the apparatus effectively excludes interpretation of this type of data, the actual temperature of the specimen being unknown.

A single specimen of Teflon was used in all measurements. It was obtained from E. I. du Pont de Nemours \& Co., Inc., and had a density of 2.219 $\mathrm{g} / \mathrm{cm}^{3}$ at $20^{\circ} \mathrm{C}$. The initial volume of the specimen was approximately $21 \mathrm{~cm}^{3}$ at $20^{\circ} \mathrm{C}$, initial volumes at other temperatures being calculated from the data of Quinn, Roberts, and Work [3], who studied a specimen cut from the same sample.

\section{Results and Discussion}

Typical compression curves of Teflon are shown in figure 1. These measurements were made at the temperatures indicated, but the curves are shown displaced along the vertical or compression axis to reduce overlapping. The numerical compression data for these curves will be given in a subsequent report because they are not of primary interest here.

It is seen in figure 1 that two transitions exist at intermediate temperatures, a high-pressure transition involving approximately a 2-percent change in volume and varying with temperature around 5,000 atm and a low-pressure transition involving a 1-percent volume change at somewhat lower pressure. This low-pressure transition occurs even at $38^{\circ} \mathrm{C}$ and at lower temperatures, but at pressures below

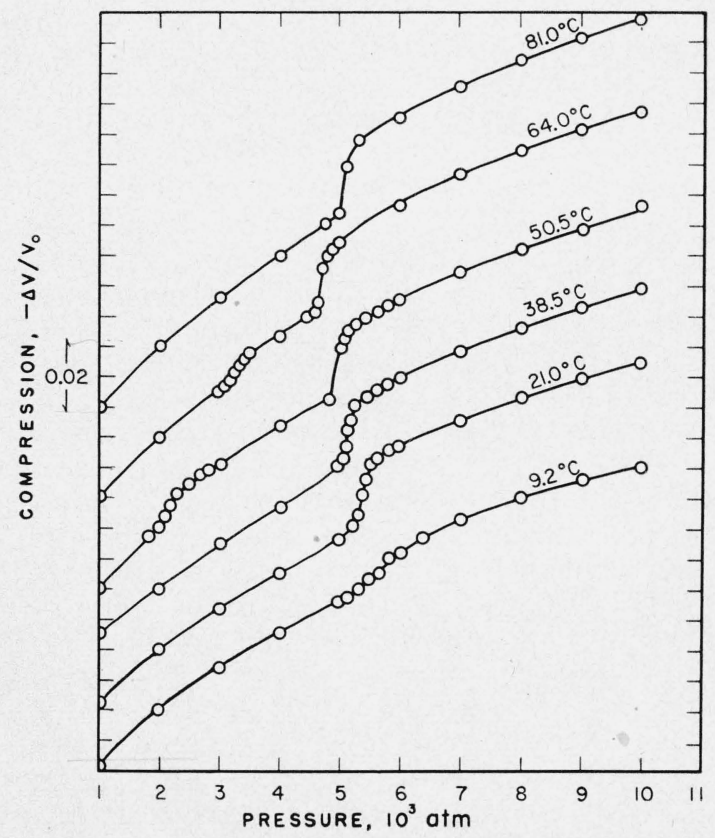

Frgure 1. Compression curves of Teflon at various temperatures.
$1,000 \mathrm{~atm}$ which is the lower limit for these data. At $81.0^{\circ} \mathrm{C}$, however, no corresponding transition can be found.

The following points of interest are to be noted in figure 1: The rounded corners at the transition pressure, combined with the existing curvature arising from pressure dependence of compressibility, render evaluation of $\Delta V$ for the transition rather uncertain. There is little doubt, however, that $\Delta V$ for the highpressure transition increases, whereas $\Delta V$ for the low-pressure transition decreases with increasing temperature.

Both transitions occur over a small pressure interval rather than at a fixed pressure. Expected small differences in structure of crystalline regions in Teflon would cause this effect as well as the rounding of the corners at the transition.

The behavior at the high-pressure transition with increasing temperature is interesting. At low temperatures this transition is "smeared out" over a rather large pressure interval, as seen in figure 1 . With increasing temperature it becomes noticeably sharper, and at temperatures above $60^{\circ} \mathrm{C}$ the transition may be observed easily, since the piston may be withdrawn 0.040 in., with no change in pressure at the transition point. This type of behavior has been referred to many times by Bridgman [10].

All data have been obtained on decreasing pressure, although there is no evidence of a region of indifference [10] in these transitions. On increasing pressure, however, with only the low-pressure phase present, considerable excess pressure must be applied to produce initiation of the transition.

Table 1. Experimental transition data on Teflon

\begin{tabular}{|c|c|c|c|c|c|}
\hline \multicolumn{6}{|c|}{ Transition } \\
\hline \multicolumn{2}{|c|}{ I to II } & \multicolumn{2}{|c|}{ II to III } & \multicolumn{2}{|c|}{ I to III } \\
\hline$P$ & $t$ & $P$ & $t$ & $P$ & $t$ \\
\hline $\begin{array}{r}\text { atm } \\
520 \\
590 \\
1,060 \\
2,130 \\
2,470 \\
3,200 \\
4,000\end{array}$ & $\begin{array}{c}{ }^{\circ} \mathrm{C} \\
29.7 \\
31.0 \\
38.5 \\
50.5 \\
57.0 \\
64.0 \\
68.5\end{array}$ & $\begin{array}{l}\text { atm } \\
5,550 \\
5,650 \\
5,540 \\
5,350 \\
5,260 \\
5,090 \\
4,910 \\
4,740 \\
4,560 \\
4,630 \\
4,590\end{array}$ & $\begin{array}{r}{ }^{\circ} \mathrm{C} \\
8.0 \\
9.2 \\
16.9 \\
21.0 \\
32.6 \\
38.6 \\
50.5 \\
57.0 \\
63.0 \\
64.0 \\
68.2\end{array}$ & $\begin{array}{l}\text { atm } \\
4.840 \\
5,050 \\
4,940\end{array}$ & $\begin{array}{r}{ }^{\circ} \mathrm{C} \\
75.0 \\
81.0 \\
81.0\end{array}$ \\
\hline
\end{tabular}

The experimental data obtained on these transitions are shown in table 1 and figure 2 . The several regions are denoted as corresponding to forms I, II, and III, and the $P, T$ data experimentally obtained on the transitions are those given in table 1.

The equilibrium line II to III is well defined and linear. The least squares equation of this line is found to be

$$
P=-17.5 \mathrm{t}+5761
$$

for pressures in atmospheres and temperatures in degrees $\mathrm{C}$. The negative slope requires in a transi- 


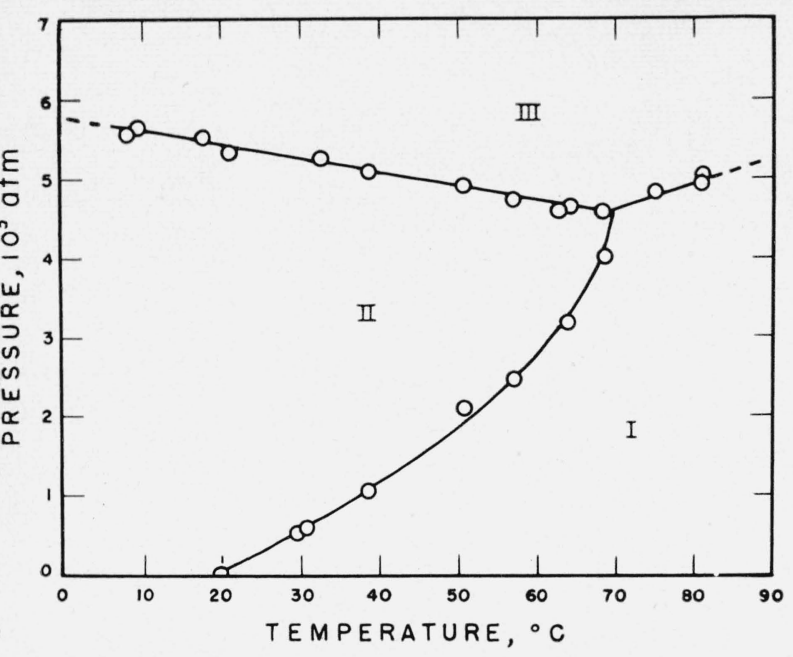

Figure 2. Proposed phase diagram for Teflon.

ion from II to III, in which the volume decreases, hat heat be absorbed. This type of transition, lthough rare under 1-atm pressure, has been bserved many times under elevated pressures [10]. Vith increasing temperature $\Delta V$ increases rather lowly along the II to III line, and from figure 1 it obvious that III is considerably less compressible han II. This difference in compressibilities has ot been evaluated quantitatively. The I to II quilibrium line is less well established but is cerainly nonlinear. Here $\Delta V$ is much smaller and ecreases rapidly with increasing temperature. ue to the decrease in $\Delta V$ with temperature, the oint shown at $68.5^{\circ} \mathrm{C}$ is subject to some uncertainty. t low pressures (that is, below $1,000 \mathrm{~atm}$ ) the xperimental points are also subject to some uncerainty, arising from the fact that the apparatus ehaves indifferently well at pressures below 700 tm. That the course of the curve is essentially orrect at low pressures, however, is shown by the greement between the latent heat of $1.54 \mathrm{cal} / \mathrm{g}$ alculated from the graphical slope at zero pressure nd the observed value of $1.91 \mathrm{cal} / \mathrm{g}$ measured by urukawa [7]. The latter value contains the total itent heat of the double transition, as mentioned reviously.

No evidence was found for any transition line riginating at the second discontinuity noted by uinn, Roberts, and Work [3] at $30^{\circ} \mathrm{C}$. The low ressures required and the small magnitude of the olume change involved would effectively obscure ny such observations with the present apparatus. nasmuch as X-ray studies have apparently disclosed no phenomena at $30^{\circ} \mathrm{C}[1,4]$, the behavior of Teflon in the vicinity of $30^{\circ} \mathrm{C}$ requires further study.

In figure 2 a triple point is shown at approximately $70^{\circ} \mathrm{C}$. That such a point exists is strongly indicated by the abrupt change in slope experienced by the II to III transition line in this region. For this reason, it is believed that a true triple point exists and that the phases I, II, and III are truly polymorphic. The alternative choice that the transitions occur in incoherent phases seems rather unlikely. It appears from the measured data that the requirement that $\Delta H=0$ for a closed path enclosing the triple point does not appear to be fulfilled. However, because the $\Delta T$ values cannot be accurately determined and $\Delta \Gamma$ for the I to II transition becomes extremely small near this point, the apparent discrepancy may not be real.

The diagram shown in figure 2 cannot represent mole than a portion of the behavior of Teflon because X-ray studies have shown melting of crystals at approximately $330^{\circ} \mathrm{C}$ [1]. At least one additional transition line must therefore appear at higher temperatures [11]. In this connection, it is to be noted that the extrapolation of the II to III transition line cuts the temperature axis at $329^{\circ} \mathrm{C}$. Inasmuch as the details of this diagram cannot be further clarified with the present apparatus, it is presented with full recognition of its lack of completeness and the fact that the proposed phases should be verified by X-ray studies.

\section{References}

[1] H. A. Rigby and C. W. Bunn, Nature 164, 583 (1949).

[2] C. E. Weir, unpublished data.

[3] F. A. Quinn, D. E. Roberts, and R. N. Work, J. Applied Phys. 22, 1085 (1951)

[4] R. H. H. Pierce, Jr., W. M. D. Bryant, and J. F. Whitney, report to the Division of Polymer Chemistry, American Chemical Society, Buffalo, N. Y., March 1952.

[5] P. W. Bridgman, Proc. Am. Acad. Arts Sci. 76, 55 (1948).

[6] C. E. Weir, J. Research NBS 46, 207 (1951) RP2192.

[7] George T. Furukawa, Robert E. McCoskey, Gerard J. King, Calorimetric properties of polytetrafluoroethylene (Teflon) from $0^{\circ}$ to $365^{\circ} \mathrm{K}$, J. Research NBS 49, 273 (1952) RP2364.

[8] C. E. Weir, J. Research NBS 45, 468 (1950) RP2160.

[9] International Critical Tables, vol. IV (McGraw-Hill Book Co., Inc., New York, N. Y., 1928).

[10] P. W. Bridgman, The physics of high pressure (G. Bell \& Sons Ltd., London, 1949).

[11] P. L. MeGeer and H. C. Duus, J. Chem. Physies 20, $1813(1952)$

Washington, November 26, 1952. 